\title{
Clinical performance of FractionLab in patient-specific quality assurance for intensity-modulated radiotherapy : a retrospective study
}

\author{
Se An $\mathrm{Oh}^{1}$, Sung Yeop Kim ${ }^{2}$, Jaehyeon Park ${ }^{1}$, Jae Won Park ${ }^{1}$, Ji Woon Yea ${ }^{1}$ \\ ${ }^{1}$ Department of Radiation Oncology, Yeungnam University College of Medicine, Daegu, Korea \\ ${ }^{2}$ Department of Physics, Yeungnam University, Gyeongsan, Korea
}

Background: This study was aimed at comparing and analyzing the results of FractionLab (Varian/Mobius Medical System) with those of portal dosimetry that uses an electronic portal imaging device. Portal dosimetry is extensively used for patient-specific quality assurance (OA) in intensity-modulated radiotherapy (IMRT).

Methods: The study includes 29 patients who underwent IMRT on a Novalis-Tx linear accelerator (Varian Medical System and BrainLAB) between June 2019 and March 2021. We analyzed the multileaf collimator DynaLog files generated after portal dosimetry to evaluate the same condition using FractionLab. The results of the recently launched FractionLab at various gamma indices (0.1\%/0.1 $\mathrm{mm}-1 \% / 1 \mathrm{~mm})$ are analyzed and compared with those of portal dosimetry $(3 \% / 3 \mathrm{~mm})$.

Results: The average gamma passing rates of portal dosimetry (3\%/3 mm) and FractionLab are 98.1\% (95.5\%-100\%) and 97.5\% $(92.3 \%-99.7 \%)$ at $0.6 \% / 0.6 \mathrm{~mm}$, respectively. The results of portal dosimetry $(3 \% / 3 \mathrm{~mm})$ are statistically comparable with the $\mathrm{QA}$ results of FractionLab (0.6\%/0.6 mm-0.9\%/0.9 mm).

Conclusion: This paper presents the clinical performance of FractionLab by the comparison of the QA results of FractionLab using portal dosimetry with various gamma indexes when performing patient-specific QA in IMRT treatment. Further, the appropriate gamma index when performing patient-specific QA with FractionLab is provided.

Keywords: Electronic portal imaging device; FractionLab; Gamma passing rate; Intensity-modulated radiotherapy; Patient-specific quality assurance

\section{Introduction}

Intensity-modulated radiotherapy (IMRT) and volumetric modulated arc therapy (VMAT), which deliver the desired radiation dose to the targeted tumors with minimal radiation to the surrounding normal tissue, are extensively used in radiotherapy. IMRT technology increases the daily radiation dose (dose per fraction) based on these technical advantages and contributes signifi- cantly to patient convenience by enhancing the radiation treatment effect and reducing the radiation treatment period [1-8]. In particular, Huh et al. [9] reported that the number of IMRT cases increased significantly by a factor of approximately 18 from 2011 to 2018 owing to the expansion of the national health insurance coverage in Korea.

IMRT modulates the photon-beam intensity by changing the position of the multileaf collimator (MLC) [10], thereby deliver-

Received: May 12, 2021 • Revised: June 22, 2021 • Accepted: August 13, 2021

Corresponding author: Ji Woon Yea, MD, PhD

Department of Radiation Oncology, Yeungnam University College of Medicine, 170 Hyeonchung-ro, Nam-gu, Daegu 42415, Korea

Tel: +82-53-620-3371•Fax: +82-53-624-3599•E-mail: yjw1160@ynu.ac.kr 
ing a nonuniform fluence from any given position of the patient's treatment beam and optimizing the dose distribution [11]. However, the calculation of the small or irregular fields frequently used in IMRT has been reported as inaccurate; even with state-of-theart dose calculation algorithms, the calculated dose distribution and the dose distribution delivered to the patient may differ [10]. Therefore, for all patients undergoing IMRT treatment, patient-specific quality assurance $(\mathrm{QA})$ must be performed prior to radiotherapy [12]. Patient-specific QA is generally analyzed using various tools, such as ion chambers, thermoluminescent dosimeters (TLDs), film dosimetry, electronic portal imaging devices (EPIDs), and two-dimensional (2D) arrays. In particular, the gamma index analysis method, which compares and analyzes the calculated and measured doses, is the most used for patient-specific QA in IMRT treatment. In IMRT treatment, although the values of acceptable dose difference (DD) and distance-to-agreement (DTA) are not clearly defined, the clinically well-accepted values are $3 \%$ and $3 \mathrm{~mm}$, respectively [10-17]. Our institution used these same values; as the passing criterion, such as DD or DTA increases, the passing gamma value will increase.

Recently, FractionLab (Varian/Mobius Medical System, Houston, TX, USA), presented a gamma index analysis of the planned and delivered fluences based on MLC log files by using a phantom-free method. FractionLab automatically analyzes the machine $\log$ files that can be generated by a medical linear accelerator. In addition, the log files can be analyzed in bulk, and several machine performance metrics, such as the MLC positioning errors, beam shutoff speed, and planned/delivered gamma agreement, can be assessed [18]. Nevertheless, the clinical performance of FractionLab has not yet been reported.

This study compared the clinical performance of FractionLab with portal dosimetry, one of the most commonly used tools for patient-specific QA in IMRT treatment. Furthermore, we attempted to determine an appropriate gamma index when performing patient-specific QA by using FractionLab.

\section{Methods}

Ethical statements: The study was approved by the Institutional Review Board (IRB) of Yeungnam University Hospital (IRB No: 2022-03-011), which waived the need for informed consent due to the retrospective design of the study.

\section{Study design and participants}

This study is a retrospective data analysis involving 29 patients who underwent IMRT on Novalis-Tx (Varian Medical System,
Palo Alto, CA and BrainLAB, Feldkirchen, Germany) linear accelerators from June 2019 to March 2021. Table 1 lists the characteristics of these patients. The treated area distribution, included in the study, shows the brain as the most commonly treated area (16 patients, 55.2\%), followed by the pelvis (six patients, 20.7\%), lung (four patients, 13.8\%), and head and neck (three patients, 10.3\%).

\section{Treatment planning and delivery techniques}

All the radiation treatments were performed using a fixed-gantry method, and the radiation was delivered using a sliding window method, in which the MLC was continuously moved during radiation exposure.

\section{Electronic portal imaging device}

Portal dosimetry (Varian Medical System) was performed for the fluences measured using an amorphous silicon (aS1000) EPID attached to the linear accelerator $[10,19]$. The EPID has a matrix of $1,024 \times 768$ pixels and detects a size of $40 \times 30 \mathrm{~cm}^{2}$ on the surface [20]. Fig. 1 shows the patient-specific QA method with the EPID in the portal dosimetry for IMRT. Portal dosimetry is extensively applied for patient-specific QA in complex radiotherapy such as IMRT and VMAT. Because portal dosimetry does not require a phantom setup, the QA time can be reduced; thus, it is widely used routinely in clinical practice. Although portal dosimetry has high resolution and reduces the QA time, it cannot verify patient dose calculation algorithms such as pencil beam convolution, anisotropic analytical algorithm, and Acuros XB algorithm. Portal dosimetry is calculated from the fluence map rather than the dose map calculation.

Fig. 2 illustrates patient-specific QA using portal dosimetry. Fig. 2 A shows the portal dose image predicted by the portal dose

Table 1. Characteristics of the studied patients treated using intensity-modulated radiotherapy techniques on the Novalis-Tx

\begin{tabular}{lc}
\hline Characteristic & Data \\
\hline No. of patients & 29 \\
Age (yr) & $66(39-87)$ \\
Sex & \\
$\quad$ Female & $5(17.2)$ \\
$\quad$ Male & $24(82.8)$ \\
Treated region & \\
$\quad$ Brain & $16(55.2)$ \\
Head and neck & $3(10.3)$ \\
Lung & $4(13.8)$ \\
Pelvis & $6(20.7)$ \\
\hline
\end{tabular}

Values are presented as number only, median (range), or number (\%). Novalis-Tx, Varian Medical System, Palo Alto, CA, USA. 


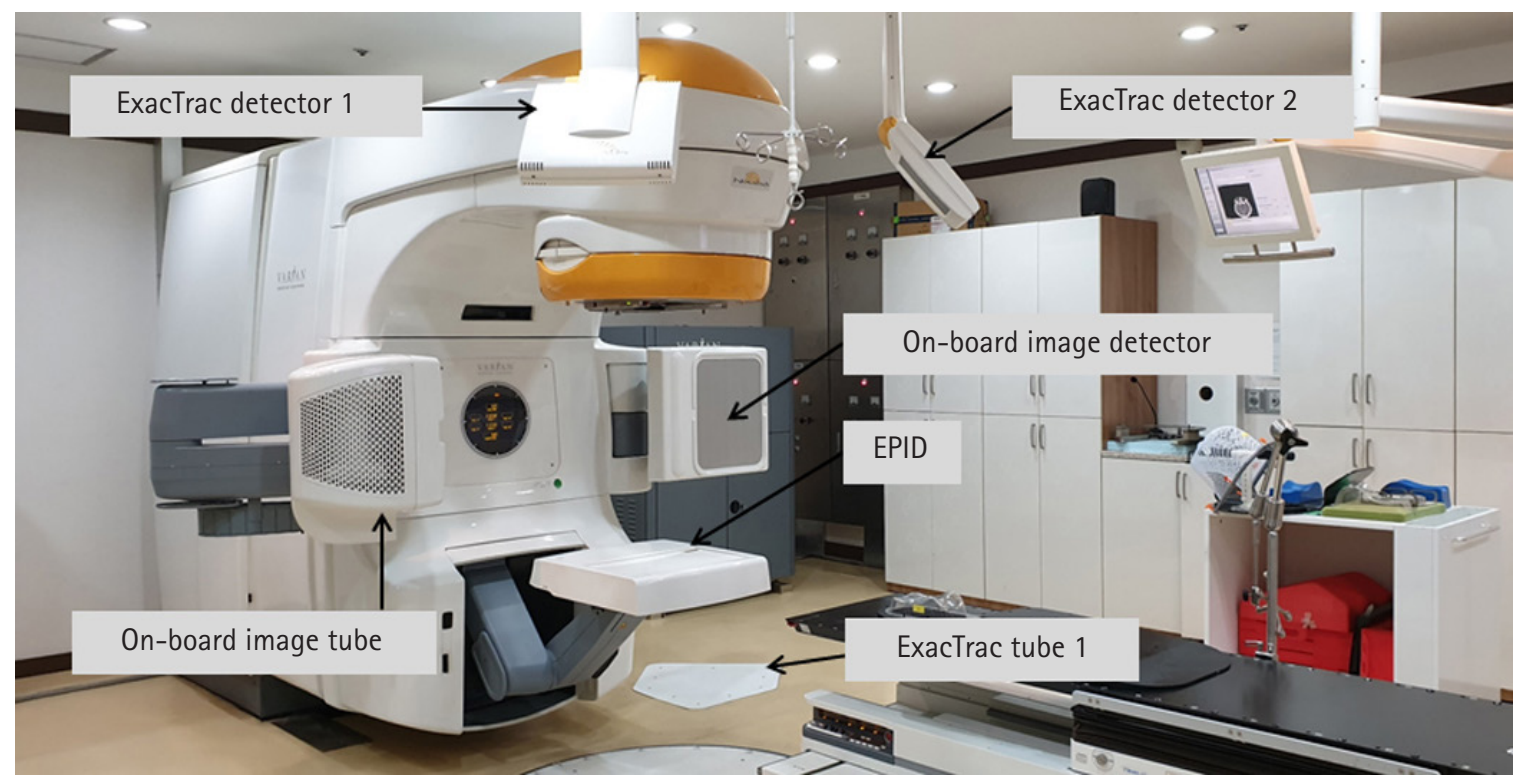

Fig. 1. Patient-specific quality assurance with amorphous silicon (aS1000) electronic portal imaging device (EPID)-based portal dosimetry.
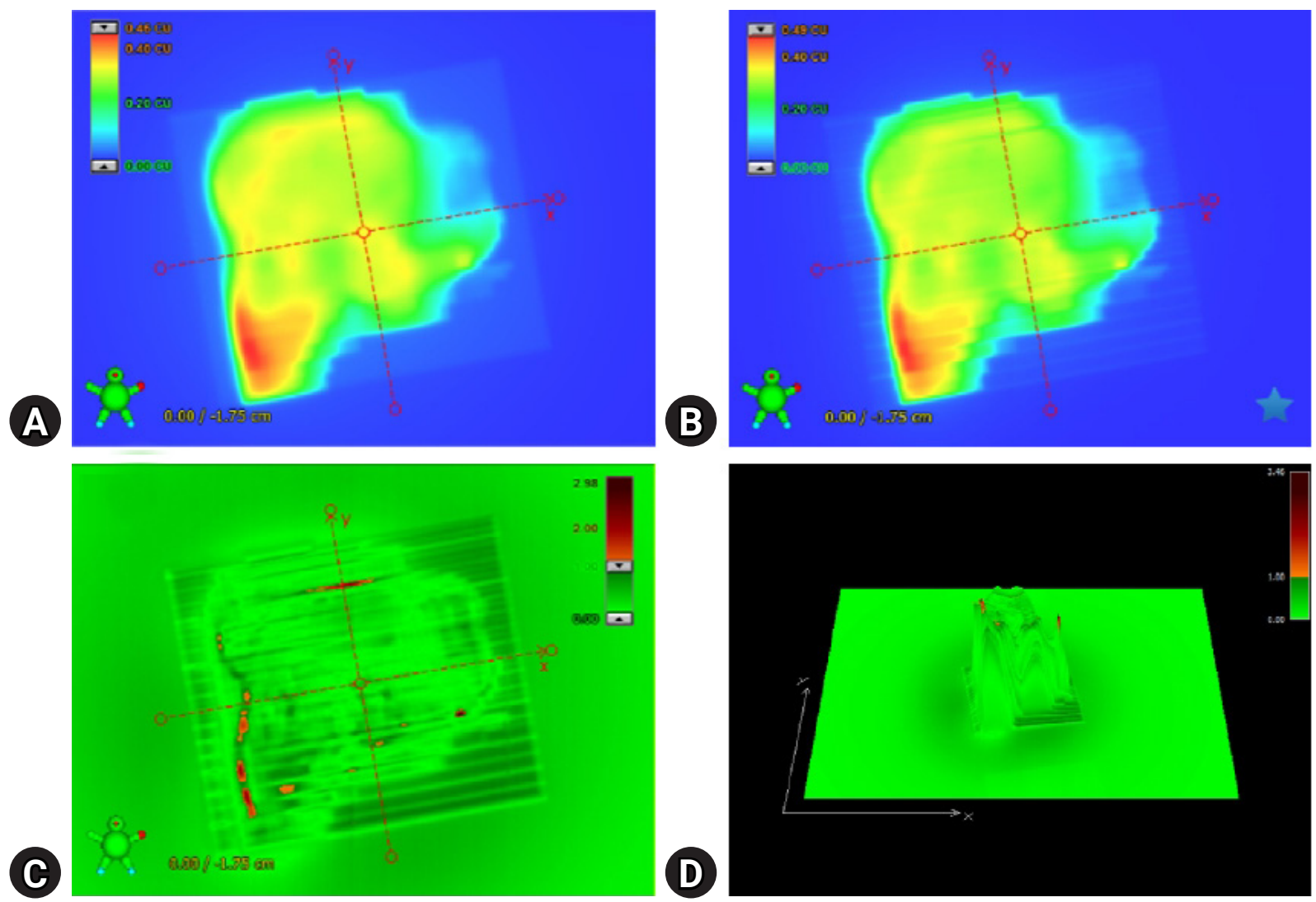

Fig. 2. Example of patient-specific quality assurance with portal dosimetry. (A) Portal dose image predicted by the portal dose image prediction (PDIP) algorithm, a dedicated two-dimensional algorithm for dose prediction. (B) Portal dose image measured by the electronic portal imaging device. (C) Gamma (3\%/3 mm) evaluation between the predicted and measured portal dose images. (D) Three-dimensional gamma $(3 \% / 3 \mathrm{~mm})$ image on the portal dose image. 
image prediction algorithm, which is a $2 \mathrm{D}$ algorithm dedicated to dose prediction, and Fig. 2B shows the image measured using the EPID. Fig. $2 \mathrm{C}$ depicts the gamma $(3 \% / 3 \mathrm{~mm})$ evaluation between the predicted and measured portal dose images, and Fig. $2 \mathrm{D}$ depicts the $3 \mathrm{D}$ gamma $(3 \% / 3 \mathrm{~mm})$ image of the portal dose image.

\section{FractionLab}

DoseLab (Varian Medical Systems) consists of three separate products: DoseLab, TG-142, and FractionLab. The FractionLab software automatically analyzes the machine log files that can be automatically generated by linear accelerators, as shown in Fig. 3.

Fig. 4 illustrates patient-specific QA using FractionLab. Fig. 4A and $4 \mathrm{~B}$ depict the planned fluence image and the fluence image delivered by the log files, and Fig. $4 \mathrm{C}$ shows the gamma (0.6\%/0.6 $\mathrm{mm}$ ) evaluation between the planned and delivered fluence images.

FractionLab performs gamma evaluation between the automatically calculated 2D fluence and the 2D fluence generated using the $\log$ files after irradiation. The machine log files include the delivered MLC position information as a function of the fractional dose, which is used by FractionLab to create fluence maps magnified on the isocenter plane. These fluence maps are generated at a fixed resolution of $0.5 \mathrm{~mm}$ per pixel [18]. Two files ('A' bank and ' $\mathrm{B}$ ' bank) were created for the machine log files of a field. FractionLab can evaluate several aspects of the machine performance such as the MLC position error, beam cutoff rate, and plan/delivery gamma agreement. DynaLog files were generated for the Varian Clinic and Varian Trilogy accelerators, and trajectory log files were generated for the Varian TrueBeam accelerators. The DynaLog files were used in this study. The general parameter specifications are as follows: sampling time $=0.05 \mathrm{sec}$, MLC position $=0.01$ $\mathrm{mm}$, jaw position $=0.1 \mathrm{~cm}$, and gantry angle $=0.1^{\circ}$; the couch an gle is not reflected in the log files [18].

\section{Analysis of the gamma index between the electronic portal imaging device and FractionLab}

Portal dosimetry was performed using a 3\% DD and 3-mm DTA, which are commonly used in clinical practice for gamma evaluation. We analyzed the MLC DynaLog files generated after portal dosimetry to evaluate the same condition using FractionLab. We evaluated the gamma value using FractionLab, by varying the DD/ DTA values from $0.1 \% / 0.1 \mathrm{~mm}$ to $1 \% / 1 \mathrm{~mm}$.

\section{Statistical analyses}

We conducted a paired $t$-test on the portal dosimetry and Fraction-

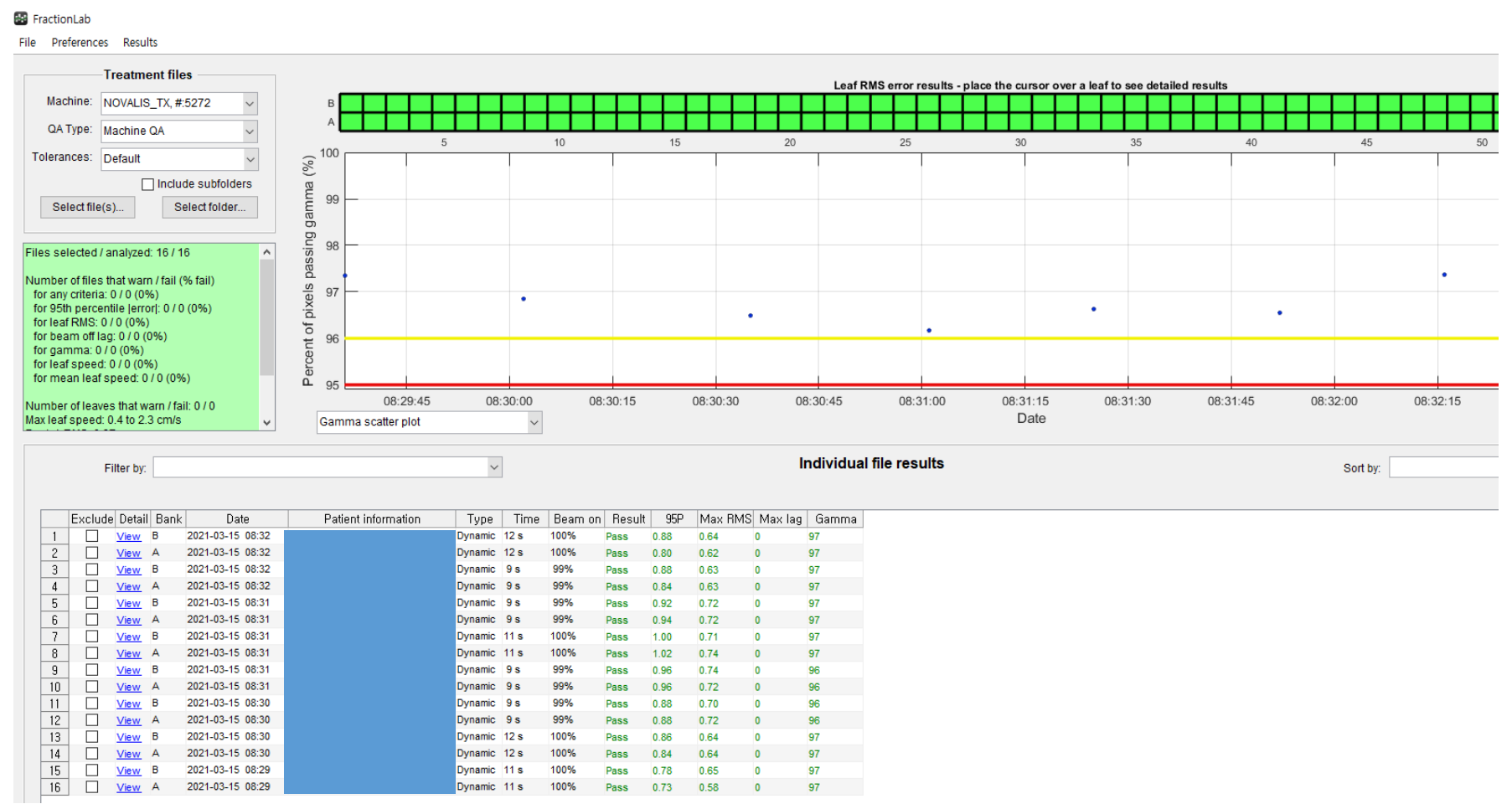

Fig. 3. Image of the gamma scatter plot by field using multileaf collimator (MLC) log files (.dlg) and FractionLab (Varian/Mobius Medical System, Houston, TX, USA), generated after the radiotherapy treatment of one of the patients included in the study; one field creates two dlg files ('A' and 'B' banks). 

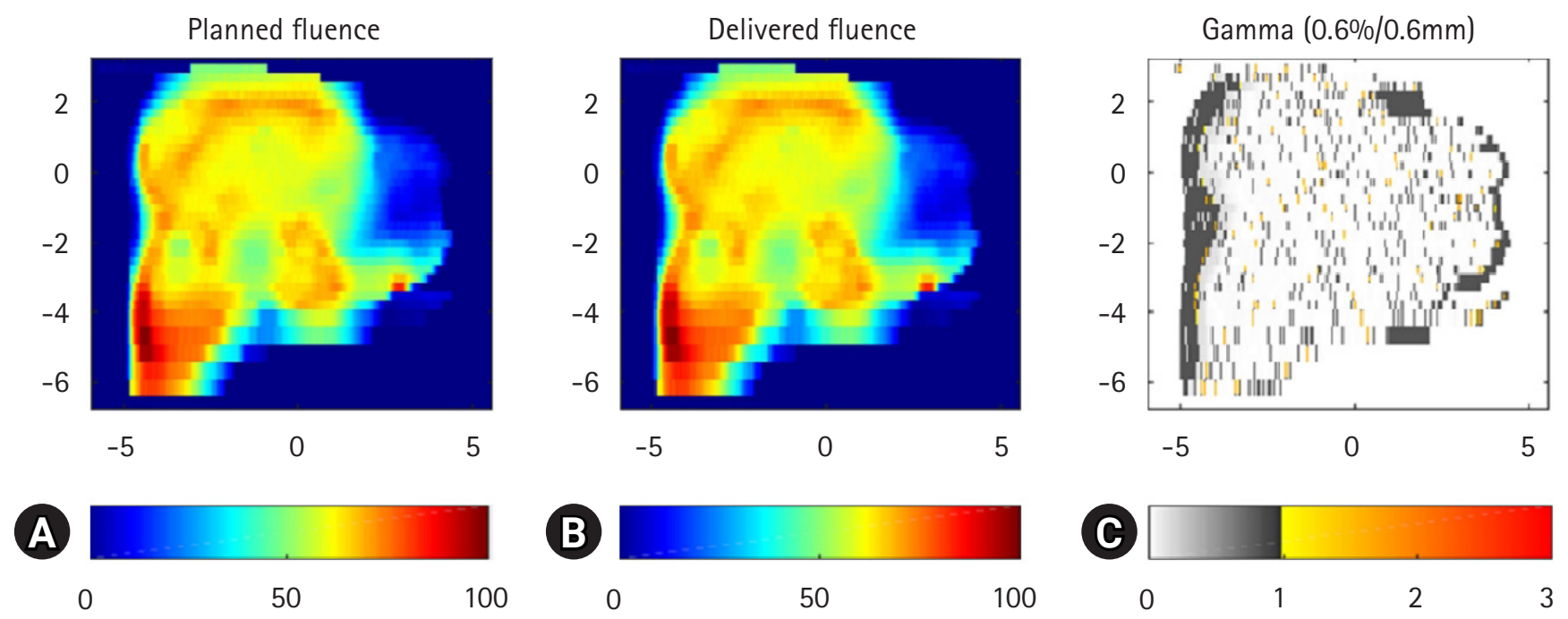

Fig. 4. Example of patient-specific quality assurance with FractionLab (Varian/Mobius Medical System, Houston, TX, USA). (A) Planned fluence image, $(B)$ Fluence image delivered by the log files, and $(C)$ gamma $(0.6 \% / 0.6 \mathrm{~mm})$ evaluation between the planned and delivered fluence images.

Lab QA results to determine an appropriate gamma index when using FractionLab-based patient-specific QA, as a 3\%/3 mm gamma index was considered when performing QA using portal dosimetry. Statistical analysis was performed using IBM SPSS version 22.0 (IBM Corp., Armonk, NY, USA). The gamma passing rates of portal dosimetry $(3 \% / 3 \mathrm{~mm})$ and FractionLab at various gamma criteria $(0.1 \% / 0.1-1 \% / 1 \mathrm{~mm})$ were analyzed, where $p \leq 0.05$ was considered statistically significant.

\section{Results}

The gamma passing rates of portal dosimetry $(3 \% / 3 \mathrm{~mm})$ and FractionLab at various gamma criteria $(0.1 \% / 0.1-1 \% / 1 \mathrm{~mm})$ for IMRT are depicted in Table 2 and Fig. 5.

The average gamma passing rate of portal dosimetry (3\%/3 $\mathrm{mm}$ ) is $98.1 \%$ (95.5\%-100\%). In FractionLab, gamma evaluation was performed from $0.1 \% / 0.1 \mathrm{~mm}$ to $1 \% / 1 \mathrm{~mm}$ in steps of $0.1 \% / 0.1 \mathrm{~mm}$. The average gamma passing rates (range) of FractionLab are $69.7 \%$ (37.7\%-77.3\%), 70.6\% (40.1\%-77.6\%), $72.4 \%$ (48.3\%-86.3\%), 74.5\% (54.5\%-94.4\%), 96.4\% (90.4\%-99.6\%), 97.5\% (92.3\%-99.7\%), 97.8\% (92.5\%-99.9\%), 98.1\% (92.8\%99.9\%), 98.5\% (93.0\%-100\%), and 99.5\% (98.1\%-100\%).

Therefore, it can be said that the paired $t$-test results for the average value of portal dosimetry $(3 \% / 3 \mathrm{~mm})$ and FractionLab exhibit statistically significant differences for gamma indices below $0.6 \% / 0.6 \mathrm{~mm}$ and $1 \% / 1 \mathrm{~mm}$.
Table 2. Gamma passing rates of portal dosimetry $(3 \% / 3 \mathrm{~mm})$ and FractionLab for various gamma criteria $(0.1 \% / 0.1-1 \% / 1 \mathrm{~mm})$ in intensity modulated radiotherapy

\begin{tabular}{lccccc}
\hline & FractionLab & & $\begin{array}{c}\text { Portal dosimetry } \\
(3 \% / 3 \mathrm{~mm})\end{array}$ & \\
\cline { 1 - 2 } \cline { 1 - 1 } $\begin{array}{c}\text { Gamma criteria } \\
(\% / \mathrm{mm})\end{array}$ & $\begin{array}{c}\text { Gamma passing } \\
\text { rates }^{\text {a }}\end{array}$ & & $\begin{array}{c}\text { Gamma passing } \\
\text { rates }^{\text {a }}\end{array}$ & \\
\cline { 1 - 2 } $0.1 / 0.1$ & $69.7(37.7-77.3)$ & & $98.1(95.5-100)$ & $<0.001$ \\
$0.2 / 0.2$ & $70.6(40.1-77.6)$ & & $98.1(95.5-100)$ & $<0.001$ \\
$0.3 / 0.3$ & $72.4(48.3-86.3)$ & & $98.1(95.5-100)$ & $<0.001$ \\
$0.4 / 0.4$ & $74.5(54.5-94.4)$ & & $98.1(95.5-100)$ & 0.001 \\
$0.5 / 0.5$ & $96.4(90.4-99.6)$ & & $98.1(95.5-100)$ & 0.001 \\
$0.6 / 0.6$ & $97.5(92.3-99.7)$ & & $98.1(95.5-100)$ & 0.127 \\
$0.7 / 0.7$ & $97.8(92.5-99.9)$ & & $98.1(95.5-100)$ & 0.519 \\
$0.8 / 0.8$ & $98.1(92.8-99.9)$ & & $98.1(95.5-100)$ & 0.965 \\
$0.9 / 0.9$ & $98.5(93.0-100.0)$ & & $98.1(95.5-100)$ & 0.306 \\
$1.0 / 1.0$ & $99.5(98.1-100.0)$ & & $98.1(95.5-100)$ & $<0.001$ \\
\hline
\end{tabular}

${ }^{\text {a) }}$ Median (range). ${ }^{\text {b) }}$ By paired $t$-test.

FractionLab, Varian/Mobius Medical System, Houston, TX, USA.

\section{Discussion}

Complex and sophisticated radiotherapy technologies, such as IMRT and VMAT, which deliver the desired radiation dose to the targeted tumors with minimum dosage to the surrounding normal organs, have a complex dose distribution and steep dose gradient. Therefore, patient-specific QA is crucial in radiotherapy [11$13,15,16]$.

Recently, various IMRT QA methods have been proposed for patient-specific QA $[10,11]$. In this study, the mean values of por- 


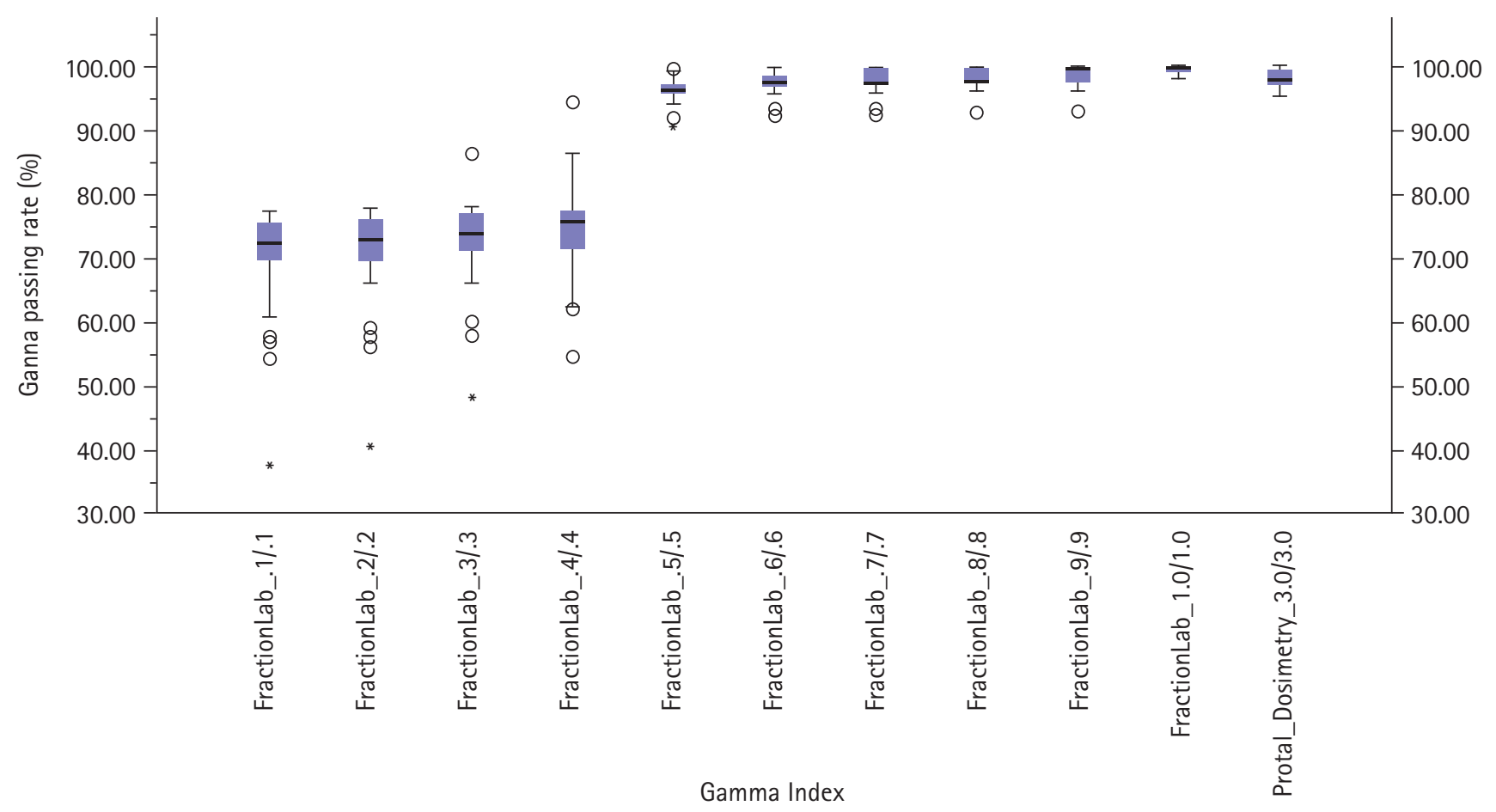

Fig. 5. Gamma passing rates of portal dosimetry $(3 \% / 3 \mathrm{~mm})$ and FractionLab (Varian/Mobius Medical System, Houston, TX, USA) at various gamma indices $(0.1 \% / 0.1 \mathrm{~mm}-1 \% / 1 \mathrm{~mm})$.

tal dosimetry $(3 \% / 3 \mathrm{~mm})$ and FractionLab (included in Dose$\mathrm{Lab}$ ) at various gamma indices were compared, and the statistical differences were analyzed through a paired $t$-test.

Kim et al. [10] investigated the characteristics of portal dosimetry in comparison with the MapCHECK2 (Sun Nuclear Corporation, Melbourne, FL, USA) measurement with respect to 65 treatment plans, including IMRT and VMAT, for various linear accelerator machines (VitalBeam, Trilogy, Clinac 21EXS, and Clinac Ix [Varian Medical System, Palo Alto, CA, USA]). When using portal dosimetry for patient-specific QA in IMRT treatment, most evaluation criteria for the gamma index include a gamma criterion of $3 \% / 3 \mathrm{~mm}$ and gamma values of $\geq 95 \%$ as pass criteria. Therefore, we analyzed the QA results by using the $3 \% / 3 \mathrm{~mm}$ gamma criteria of portal dosimetry and logfiles generated by irradiation in portal dosimetry using FractionLab with various gamma indices. We tried to find an appropriate gamma index when performing patient-specific QA with FractionLab using the QA results of portal dosimetry. The results showed that performing gamma index in the range of $0.6 \% / 0.6 \mathrm{~mm}$ and $0.9 \% / 0.9 \mathrm{~mm}$ is appropriate if FractionLab is used for patient-specific QA in IMRT.

Patient-specific QA has been conventionally performed using a phantom-based system with various QA tools such as ion chambers, TLDs, film dosimetry, EPID, and 2D arrays. However, the use of such a phantom-based QA is time-consuming and the dose per fraction delivered cannot be tracked. In addition, this method is incapable of determining the root cause of failures. Moreover, the conventional method ignores patient-specific anatomical variations. In comparison, a logfiles-based QA system, such as Mobius3D (Varian Medical System) and ArcCHECK (Sun Nuclear), will enable automation, tracking of heterogeneous anatomical dose, and allow for root-cause analysis [21].

As no clinical data on patient-specific QA using FractionLab are currently available, the clinical results of this study can be useful for medical physicists in radiation oncology.

However, this study has some limitations. First, we only analyzed the clinical performance of the two systems by using the gamma analysis method based on portal dosimetry and FractionLab, and did not provide a detailed analysis of the algorithms of the two systems. Second, this study included only fixed-gantry IMRT patients. More complex radiotherapy, such as VMAT, may produce different results. In the future, it would be necessary to compare the existing patient-specific QA methods for treatments such as VMAT and various linear accelerators.

This study showed the clinical performance of FractionLab by comparing its QA results using portal dosimetry and various gamma indexes with the results of patient-specific QA in IMRT treatment. The proposed method can present the appropriate gamma index when performing patient-specific QA with FractionLab. In 
patient-specific QA of IMRT treatment, the QA result using a gamma index of $3 \% / 3 \mathrm{~mm}$ using portal dosimetry is considered interchangeable with the QA result obtained using a gamma index in the range of $0.6 \% / 0.6 \mathrm{~mm}$ and $0.9 \% / 0.9 \mathrm{~mm}$ of FractionLab.

\section{Notes}

\section{Conflicts of interest}

No potential conflict of interest relevant to this article was reported.

\section{Funding}

This work was supported by the National Research Foundation of Korea (NRF) grant funded by the Korean government (MSIT) (No. 2021R1G1A1003209).

\section{Author contributions}

Conceptualization: SAO, SYK, JP, JWP, JWY; Data curation, Formal analysis: SYK, JWY; Funding acquisition: SAO; Methodology: SYK, JP, JWP; Investigation: SAO, SYK; Validation: JP, JWP; Project administration, Software, Supervision: JWY; Writing original draft: SAO; Writing - review \& editing: SAO, JP, JWP, JWY.

\section{ORCID}

Se An Oh, https://orcid.org/0000-0002-8835-2814

Sung Yeop Kim, https://orcid.org/0000-0002-2921-6126

Jaehyeon Park, https://orcid.org/0000-0003-2546-4808

Jae Won Park, https://orcid.org/0000-0003-1100-5301

Ji Woon Yea, https://orcid.org/0000-0002-7699-342X

\section{References}

1. Dai X, Zhao Y, Liang Z, Dassarath M, Wang L, Jin L, et al. Volumetric-modulated arc therapy for oropharyngeal carcinoma: a dosimetric and delivery efficiency comparison with static-field IMRT. Phys Med 2015;31:54-9.

2. Ali M, Babaiah M, Madhusudhan N, George G. Comparative dosimetric analysis of IMRT and VMAT (RapidArc) in brain, head and neck, breast and prostate malignancies. Int J Cancer Ther Oncol 2015;3:03019.

3. Mani KR, Upadhayay S, Das KJ. Influence of jaw tracking in intensity-modulated and volumetric-modulated arc radiotherapy for head and neck cancers: a dosimetric study. Radiat Oncol J 2017;35:90-100.

4. Liu X, Huang E, Wang Y, He Y, Luo H, Zhong M, et al. Dosimetric comparison of helical tomotherapy, VMAT, fixed-field
IMRT and 3D-conformal radiotherapy for stage I-II nasal natural killer T-cell lymphoma. Radiat Oncol 2017;12:76.

5. Deng X, Han C, Chen S, Xie C, Yi J, Zhou Y, et al. Dosimetric benefits of intensity-modulated radiotherapy and volumetric-modulated arc therapy in the treatment of postoperative cervical cancer patients. J Appl Clin Med Phys 2017;18:25-31.

6. Mellon EA, Javedan K, Strom TJ, Moros EG, Biagioli MC, Fernandez DC, et al. A dosimetric comparison of volumetric modulated arc therapy with step-and-shoot intensity modulated radiation therapy for prostate cancer. Pract Radiat Oncol 2015; 5:11-5.

7. Oh SA, Yea JW, Park JW, Park J. Use of a head-tilting baseplate during volumetric-modulated arc therapy (VMAT) to better protect organs at risk in hippocampal sparing whole brain radiotherapy (HS-WBRT). PLoS One 2020;15:e0232430.

8. Oh SA, Kang MK, Kim SK, Yea JW. Comparison of IMRT and VMAT techniques in spine stereotactic radiosurgery with international spine radiosurgery consortium consensus guidelines. Prog Med Phys 2013;24:145-53.

9. Huh SJ, Park W, Choi DH. Recent trends in intensity-modulated radiation therapy use in Korea. Radiat Oncol J 2019;37:24953.

10. Kim JI, Choi CH, Park SY, An H, Wu HG, Park JM. Gamma evaluation with portal dosimetry for volumetric modulated arc therapy and intensity-modulated radiation therapy. Prog Med Phys 2017;28:61-6.

11. Yea JW, ParkJW, Kim SK, Kim DY, Kim JG, Seo CY, et al. Feasibility of a 3D-printed anthropomorphic patient-specific head phantom for patient-specific quality assurance of intensity-modulated radiotherapy. PLoS One 2017;12:e0181560.

12. Ezzell GA, Burmeister JW, Dogan N, LoSasso TJ, Mechalakos JG, Mihailidis D, et al. IMRT commissioning: multiple institution planning and dosimetry comparisons, a report from AAPM Task Group 119. Med Phys 2009;36:5359-73.

13. Oh SA, Yea JW, Lee R, Park HB, Kim SK. Dosimetric verifications of the output factors in the small field less than $3 \mathrm{~cm} 2$ using the Gafchromic EBT2 films and the various detectors. Prog Med Phys 2014;25:218-24.

14. Oh SA, Kang MK, Yea JW, Kim SH, Kim KH, Kim SK. Comparison of intensity modulated radiation therapy dose calculations with a $\mathrm{PBC}$ and $\mathrm{AAA}$ algorithms in the lung cancer. Korean J Med Phys 2012;23:48-53.

15. Olaciregui-Ruiz I, Vivas-Maiques B, Kaas J, Perik T, Wittkamper F, Mijnheer B, et al. Transit and non-transit 3D EPID dosimetry versus detector arrays for patient specific QA. J Appl Clin Med Phys 2019;20:79-90.

16. Han B, Ding A, Lu M, Xing L. Pixel response-based EPID do- 
simetry for patient specific QA.J Appl Clin Med Phys 2017;18: 9-17.

17. Defoor DL, Stathakis S, Roring JE, Kirby NA, Mavroidis P, Obeidat M, et al. Investigation of error detection capabilities of phantom, EPID and MLC log file based IMRT QA methods. J Appl Clin Med Phys 2017;18:172-9.

18. Varian Medical Systems. DoseLab user guide - version 7.0 [Internet]. Houston, TX: Varian Medical Systems; 2018 [cited 2021 May 6]. https://www.myvarian.com/s/productdocumentation?lang $=$ en.

19. Kim YL, Chung JB, Kim JS, Lee JW, Choi KS. Comparison of the performance between portal dosimetry and a commercial two-dimensional array system on pretreatment quality assurance for volumetric-modulated arc and intensity-modulated radiation therapy.J Korean Phys Soc 2014;64:1207-12.

20. Krishna Murthy K. Patient-specific quality assurance of RapidArc treatments: portal prediction dosimetry compared with phantom studies. Biomed Imaging Interv J 2012;8:e28.

21. Stanhope CW, Drake DG, Liang J, Alber M, Söhn M, Habib C, et al. Evaluation of machine log files/MC-based treatment planning and delivery QA as compared to ArcCHECK QA. Med Phys 2018;45:2864-74. 